\title{
Patients who wore standard magnetic bracelets reported reduced pain from osteoarthritis of the hip or knee compared with patients wearing placebo bracelets
}

Harlow T, Greaves C, White A, et al. Randomised controlled trial of magnetic bracelets for relieving pain in osteoarthritis of the hip and knee. BMJ 2004;329:1450-4.

Does short term use of magnetic bracelets reduce pain in patients with osteoarthritis of the hip and knee?

\section{METHODS}

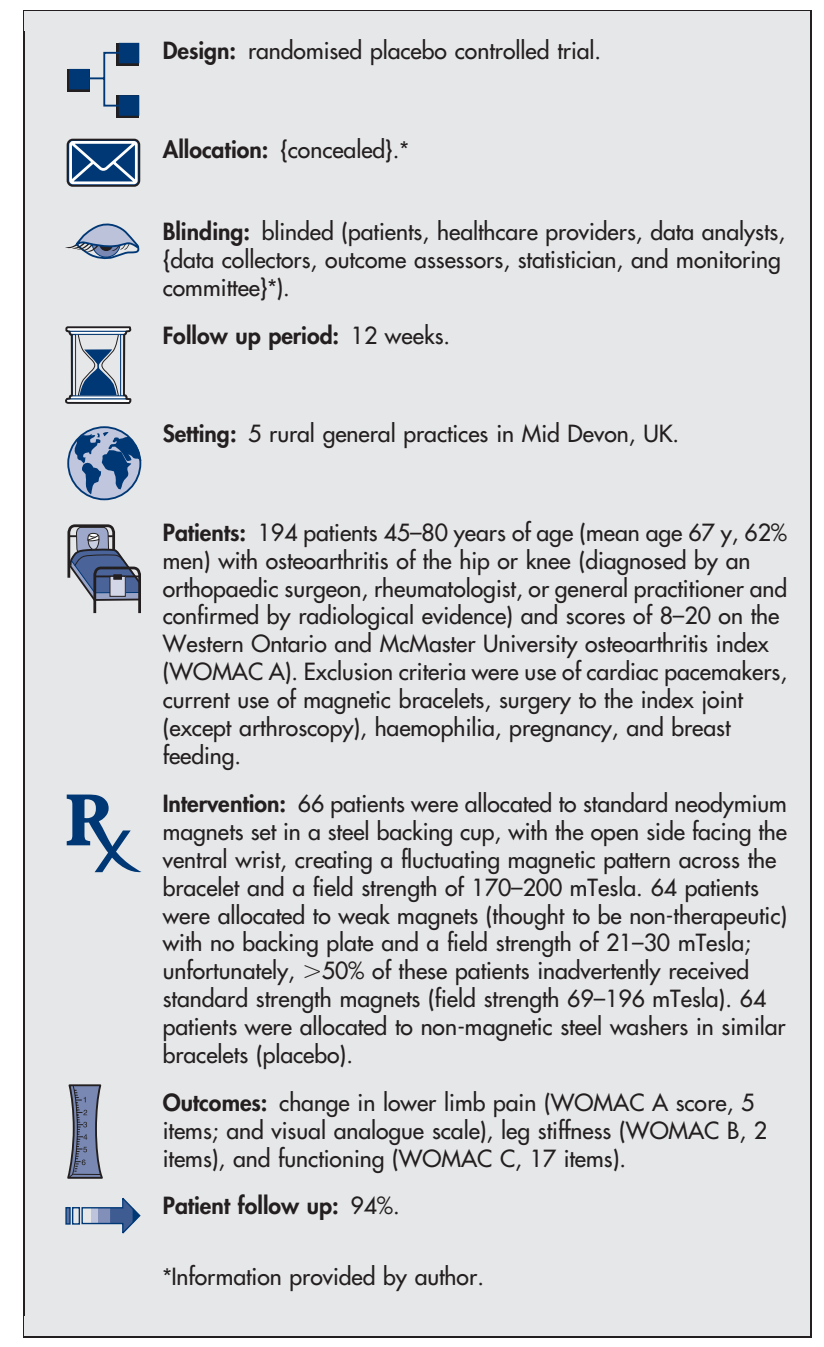

\section{MAIN RESULTS}

At 12 weeks, the standard magnet group had greater pain reduction (mean difference in WOMAC A scores 1.3 points, $95 \%$ CI 0.09 to 2.60 ) and improvement in functioning (mean difference in WOMAC C scores 4.4, CI 1.0 to 7.9 ) than the placebo group, but did not differ from the weak magnet group for either of these outcomes (mean difference in WOMAC A scores 0.81 , CI -0.44 to 2.07 , and mean difference in WOMAC C scores $3.3, \mathrm{CI}-0.2$ to 6.7 ). The 3 groups did

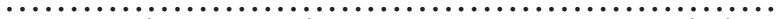
For correspondence: Dr T Harlow, Hospiscare, Exeter, UK. timharlow@ eclipse.co.uk

Source of funding: Arthritis Research Campaign. not differ for change in stiffness scores assessed by WOMAC B scores $(\mathrm{p}=0.48)$.

\section{CONCLUSION}

Patients who wore standard magnetic bracelets reported reduced pain associated with osteoarthritis of the hip and knee at 12 weeks compared with patients who wore placebo bracelets.

Commentary

The study by Harlow et al contributes to the literature on use of magnets for chronic pain but fails to provide conclusive evidence about their effectiveness. Because of the widespread use of magnets, nurses need to be aware of these findings.

Despite using an innovative study design that used "weak" magnets to provide an undetectable placebo, the study by Harlow et al failed to fully blind study participants and failed to show an effect of undisputable importance. In terms of blinding, $54 \%$ of patients in the standard magnet group and $47 \%$ of patients in the placebo magnet group correctly guessed their magnet type. However, it is not known whether their "correct" guesses went beyond what would be expected by chance. Successful blinding has been difficult to achieve in studies involving magnets because of the easily detectable magnetic force. In this study, the use of weak magnets failed largely because of a manufacturing error, which resulted in wide variability in the strength of weak magnets, with some having field strengths in the same range as standard magnets. Thus the usefulness of this blinding technique is not known.

With respect to the estimates of effect for pain reduction and improvement in functioning, the lower boundaries of the confidence intervals (0.09 and 1.0, respectively) represent small differences between groups and indicate that the trial is non-definitive. In addition, this trial involved a sample of predominantly white people with fairly severe osteoarthritis (WOMAC A score $\geqslant 8$ ) who also took painkillers 5-7 days per week. Larger studies, involving more rigour in magnet manufacturing and conducted on individuals from various ethnic backgrounds, are needed to overcome these weaknesses.

The lack of evidence on magnet effectiveness dictates that nurses should neither recommend nor discourage their use but rather should provide education to clients. In one study of 146 patients with arthritis, almost $30 \%$ used copper or magnetic bracelets. ${ }^{1}$ Although magnets are usually promoted as being safe and inexpensive, several contradictions to use have been described, including pregnancy (because of the unknown effect on foetuses) and patients with cardiac pacemakers, defibrillators, or insulin pumps (because these devices are magnetically controlled). ${ }^{2}$ Additionally, magnets sold to consumers have variable composition, strength, and prices. Nurses have an important role in promoting health literacy about the use of magnets for chronic pain.

Heather L McDonald, RN, MSc University of British Columbia Vancouver, British Columbia, Canada

1 Rao JK, Mihaliak K, Kroenke K, et al. Use of complementary therapies for arthritis among patients of rheumatologists. Ann Intern Med 1999;131:409-16.

2 Ratterman R, Secrest J, Norwood B, et al. Magnet therapy: what's the attraction? J Am Acad Nurse Pract 2002;14:347-53. 